\title{
ERRATUM
}

\section{Erratum to: Superconductivity and spin fluctuations}

\author{
Shiliang $\mathbf{L i}^{1, \dagger}$, Pengcheng Dai ${ }^{1,2, \ddagger}$ \\ ${ }^{1}$ Beijing National Laboratory for Condensed Matter Physics, Institute of Physics, \\ Chinese Academy of Sciences, Beijing 100190, China \\ ${ }^{2}$ Department of Physics and Astronomy, The University of Tennessee, Knoxville, TN 37996-1200, USA \\ Corresponding authors. E-mail: ${ }^{\dagger}$ slli@aphy.iphy.ac.cn, ${ }^{\ddagger} p d a i @ u t k . e d u$
}

ERRATUM TO: Front. Phys. 6(4), 429 (2011), doi:10.11 07/s11467-011-0221-0

In the original publication of this article the DOI number is incorrect. It should be doi:10.1007/s11467-0110221-0.
The online version of the original article can be found at https://doi.org/10.1107/s11467-011-0221-0 and http:// journal.hep.com.cn/fop/EN/10.1107/s11467-011-0221-0.

\footnotetext{
*This article can also be found at http://journal.hep.com. $\mathrm{cn} /$ fop/EN/10.1007/s11467-020-1033-x.
} 